Resa Ocio, A. (2021). La formación en igualdad de género en los grados de Educación Primaria. Revista Electrónica Interuniversitaria de Formación del Profesorado, 24(1), 13-27.

\title{
La formación en igualdad de género en los grados de Educación Primaria
}

\author{
Ainhoa Resa Ocio \\ Universidad Complutense de Madrid
}

\section{Resumen}

El artículo a continuación versa sobre la necesidad de adquirir una perspectiva de género para alcanzar la igualdad real en la escuela. La legislación respalda la igualdad de género como un principio de la educación. A fin de comprobar si la formación inicial del profesorado es adecuada a este respecto, se realiza un análisis de los planes de estudios de los grados de Educación Primaria desde una óptica de género. Para ello, se realiza una aproximación cualitativa basada en el análisis en profundidad del contenido de 72 guías docentes de los grados de Educación Primaria de la Universidad Complutense de Madrid y de entrevistas realizadas al profesorado. Los resultados muestran cómo la formación inicial del profesorado de los grados de Educación Primaria continúa girando en torno a una visión androcéntrica de la educación, mantiene un lenguaje sexista, un currículum en el que se invisibiliza a las mujeres que no se ajusta a la legislación vigente y no garantiza que las futuras generaciones sean educadas en igualdad. Se abre así la posibilidad de explorar la formación en igualdad de género a un nivel más amplio y la investigación sobre la aplicación efectiva de la coeducación en el sistema educativo español.

\section{Palabras clave}

Coeducación; Género; Formación inicial del profesorado; Igualdad; Educación Primaria.

\section{Gender equality training in Primary Education degrees}

\begin{abstract}
The article above deals with the need to acquire a gender perspective so as to achieve true equality in a school setting. This legislation backs gender equality as a principle of education. In order to confirm if the initial teacher training is adequate in this respect, an analysis of the curricula of the education degrees is conducted with a gender viewpoint. For
\end{abstract}


this purpose, a qualitative approximation based on the analysis of content from 72 teaching guides and interviews with university teachers was carried out. The results show how teacher training in Primary Education degrees still revolves around an androcentric view of education, this has not adjusted to current legislation. Therefore, this does not guarantee that future generations are correctly educated in equality. This opens the possibility of exploring gender equality training at a broader level and research on the effective application of coeducation in the Spanish educational system.

\section{Key words}

Coeducation; Gender; Teacher training; Equality; Primary education.

\section{Introducción}

La educación es un medio de producción, reproducción y legitimación de la cultura y las relaciones de poder (Bourdieu y Passeron, 1996). En la escuela se perpetúan las desigualdades y se favorece su continuación (Gómez, 2015) pero, al mismo tiempo, se abre la posibilidad del cambio y la mejora de la sociedad. En las últimas décadas se ha venido dando un gran avance con respecto a la igualdad de género en la sociedad española, pero esta perspectiva aún ha de extenderse a todos los ámbitos e instituciones y, de manera específica, al contexto educativo. La legislación en materia educativa establece la igualdad como una obligatoriedad en los centros educativos.

El profesorado constituye una variable clave en la igualdad ya que es su misión formar a las jóvenes generaciones. Desde esta perspectiva, es imperativo formar al futuro profesorado desde y hacia una educación igualitaria, democrática y de calidad. En España, desde la aprobación de la Ley Orgánica 3/2007, de 22 de marzo, para la igualdad efectiva de mujeres y hombres, debe introducirse formación específica en la formación inicial y permanente del profesorado. Sin embargo, casi una década después, Cabeza y Rodríguez (2015) todavía alertan de la existencia de una desconexión entre la teoría y la práctica:

La Agencia Nacional de Evaluación de la Calidad y Acreditación (ANECA) es la responsable de revisar y vigilar que se cumplan las recomendaciones de la Ley de Igualdad y la inclusión de las asignaturas de género en los nuevos planes de estudio, pero en la práctica esto no está ocurriendo y estas asignaturas, salvo excepciones, se están quedando fuera de estos planes. (p.40).

En este contexto, el objetivo del presente artículo es explorar en qué medida la formación inicial del profesorado, de manera específica y transversal, responde a la demanda de integrar la igualdad de género en sus planes de estudios.

\section{La coeducación en España: una revisión histórica}

La Escuela Moderna de Ferrer i Guàrdia, la Escuela Nueva y la Institución Libre de Enseñanza fueron los movimientos precursores del modelo educativo de la Segunda República. Durante este periodo, se unificaron los espacios y currículos de niñas y niños. Se siguió un modelo coeducativo cuyo trasfondo ideológico se basaba en la búsqueda de la igualdad y el cuestionamiento de los roles de género. El modelo coeducativo se refiere al "proceso intencionado de intervención a través del cual se potencia el desarrollo de niños y niñas partiendo de la realidad de dos sexos diferentes hacia un desarrollo personal y una construcción social comunes y no enfrentados" (Colectivo Feminario de Alicante, 1987). 
Subirats (1991) entiende que la coeducación ha de tratar de solventar los comportamientos que sustentan la discriminación de las mujeres. Para ello, Rebollo (2006) sugiere que en este modelo cobran valor las visiones, valores y actitudes tradicionalmente asociados a las mujeres y se eliminan los estereotipos y relaciones de poder. Este modelo, que fue criticado por los sectores más conservadores y la Iglesia, se desechó durante el franquismo (Ballarín, 2001).

La legislación franquista prohibió la escolarización conjunta de niñas y niños mientras que la formación de las chicas quedó a cargo de la Sección Femenina de la Falange. Se volvió a un modelo de escuela segregada ${ }^{1}$ según el cual, como apunta Rebollo (2006), se educa a cada individuo para el desarrollo de su rol sexual respectivo a través de la separación física en escuelas diferentes y la adaptación de la metodología y el currículum de acuerdo a diferencias atribuidas como naturales de cada sexo. La Ley General de Educación de 1970 (LGE) supuso un primer acercamiento a una escuela más igualitaria puesto que retomaba un modelo mixto que si bien suponía compartir espacios comunes y un currículum unificado, se trataba de una igualdad de carácter únicamente formal (ibíd., 2008).

Durante el proceso de redacción de la Constitución de 1978, la Plataforma Feminista de Madrid hizo especial hincapié en la inclusión de un artículo que recogiera lo siguiente:

“El estado sólo protegerá la enseñanza estatal, que será laica, mixta, gratuita y obligatoria. A tal efecto, el estado garantizará que se realice sin discriminación o menoscabo por razón de sexo, implantando la coeducación efectiva a todos los niveles y sancionando a los establecimientos que no cumplieran con este principio" (Varela, 2014) $)^{2}$.

A pesar de que este artículo no fue aceptado, sí se eliminó la separación física y las diferencias en el currículum entre las chicas y los chicos. Sin embargo, el paso de la escuela segregada a la escuela mixta no combatía la desigualdad de género en la escuela. De hecho, como sugiere Ballarín (2008), la escuela mixta legitima y naturaliza las desigualdades convirtiendo a las mujeres en personas sumisas y a los hombres en dominadores.

Años más tarde, con la Ley Orgánica 1/1990, de Ordenación General del Sistema Educativo (LOGSE), se abogó por replantear la educación desde la perspectiva de igualdad de género. Así, además de rechazar la discriminación sexual, los prejuicios, los estereotipos de género y el lenguaje sexista, se reconocían y valoraban los diferentes roles de género y se impulsaba la coeducación en el marco de la familia y la escuela (Azorín, 2015).

Posteriormente, las Leyes sucesivas Ley Orgánica de Calidad de la Educación (LOCE), Ley Orgánica de Educación (LOE) y Ley Orgánica para la Mejora de la Calidad Educativa (LOMCE), han puesto de manifiesto la evolución social que se ha dado en este terreno ya que al tratar la igualdad de género explicitan la necesidad de atender a la misma, estableciendo así los pilares que han de sustentar la educación actual.

\footnotetext{
1 Los términos educación separada, segregada y diferenciada aun siendo sinónimos tienen connotaciones diferentes. Subirats (2010) presenta el término escuela diferenciada como aquel que proponen algunas de las personas a favor de este modelo educativo mientras que educación segregada es el habitual entre las detractoras del mismo.

2 La Constitución, en su artículo 27 de los derechos y deberes fundamentales, establece que: 1. Todos tienen el derecho a la educación. Se reconoce la libertad de enseñanza. 2. La educación tendrá por objeto el pleno desarrollo de la personalidad humana en el respeto a los principios democráticos de convivencia y a los derechos y libertades fundamentales. 3. Los poderes públicos garantizan el derecho que asiste a los padres para que sus hijos reciban la formación religiosa y moral que esté de acuerdo con sus propias convicciones. 4 . La enseñanza básica es obligatoria y gratuita.
} 
Al margen de la legislación educativa, la Ley Orgánica 1/2004, de 28 de diciembre, de medidas de Protección Integral contra la Violencia de Género, en su capítulo I establece una regulación en todos los niveles educativos y acciones concretas en cuanto a la formación del profesorado tanto inicial como permanente a través de formación específica en materia de igualdad. La Ley Orgánica 3/2007, de 22 de marzo, para la igualdad efectiva de mujeres y hombres, en su artículo 24, decreta la integración del principio de igualdad en la política de educación. Esto se materializa en principios y actuaciones concretas tales como la atención a la igualdad en los currículos, el reconocimiento de la mujer en la historia o la eliminación de los comportamientos y contenidos sexistas y los estereotipos. Además, aparece la obligación del estudio y la aplicación de la igualdad en la formación inicial y permanente del profesorado.

\section{La formación inicial del profesorado en igualdad de género}

La formación del profesorado es un tema complejo puesto que, en el desempeño de su labor docente, "no sólo enseñan conocimientos instrumentales, sino también aportan formación ética, cultural, social, afectiva, etc." (González, 2017). En España, la formación del futuro profesorado de Educación Primaria se ofrece en las universidades. Magisterio se introdujo en la universidad en el año 1970, con la LGE y, si bien supuso la renovación de espacios, manuales y materiales didácticos, estos cambios no fueron suficientes para modificar mentalidades y comportamientos estancos (Escolano, 2002 citado en González, 2017, p. 171). En España, la preocupación por integrar la perspectiva de género en la docencia universitaria no ha comenzado a recibir una atención significativa hasta los últimos años (Ortega, 2018). Con la implantación de las anteriormente citadas Ley Orgánica de Medidas de Protección Integral contra la Violencia de Género (2004) y Ley de Medidas para la Igualdad Efectiva de Mujeres y Hombres (2007), la igualdad de género debe introducirse en el currículum. A pesar de ello, según Díaz de Greñu y Anguita (2017), la escuela todavía sigue un modelo sexista en la toma de decisiones sobre contenidos, la aplicación de las estrategias metodológicas, la selección de los recursos didácticos, las relaciones interpersonales, la forma de distribución del espacio y el reparto de tareas.

Así, la formación en igualdad de género y diversidad sexual en Educación Primaria difiere según las Comunidades Autónomas. A modo de ejemplo, en Andalucía y Cataluña es obligatoria la existencia de una persona responsable de coeducación y la inclusión de la perspectiva de género en el Plan de Desarrollo del centro. En otras comunidades, sin embargo, no se contempla ninguna actuación al respecto o bien se han puesto en marcha programas de coeducación voluntarios para los centros dotados de escaso presupuesto (Gómez y Sánchez, 2017). La Comunidad de Madrid, a través del DECRETO 89/2014, de 24 de julio, establece el Currículo de la Educación Primaria y concreta que, además de integrarse los contenidos necesarios para educar en igualdad de género, estos han de ser transversales al currículum (Art.8,2). La ley 2/2016, de 29 de marzo, de Identidad y Expresión de Género e Igualdad Social y no Discriminación de la Comunidad de Madrid, en su artículo 25 , decreta, además, que los cursos de formación del profesorado han de incorporar la diversidad sexual y de género, así como fomentar el abordaje en el aula de la igualdad de género.

La Universidad Complutense de Madrid, desde el año 2017, recoge también en el artículo 2 de su estatuto (DECRETO 32/2017) que la institución vela por la no discriminación por cualquier circunstancia, ya sea de carácter personal o social, incluyendo de manera explícita el sexo. 


\section{Metodología}

Para analizar en qué medida la formación del profesorado incluye cuestiones relacionadas con la igualdad de género se ha realizado una doble aproximación metodológica. Por un lado, se han analizado 72 guías de las asignaturas del curso 2018-2019 del Grado Maestro en Educación Primaria, incluidas las del Practicum y Trabajo Final de Grado. Si bien se ha tratado de acceder a los programas específicos del profesorado que imparte las asignaturas para realizar un análisis más exhaustivo, no ha sido posible ya que el profesorado que ha respondido a la solicitud ha afirmado que su referente en su práctica docente son las guías de las asignaturas que aparecen publicadas en la página web de la universidad.

Para el análisis de las guías, se ha tomado como referente el instrumento diseñado por Ros et al. (2016), adaptado a las necesidades y contexto de la investigación, que consiste en tres categorías: lenguaje inclusivo, contenidos coeducativos y revisión de la bibliografía. Este modelo se ha complementado con indicadores de elaboración propia, cuyos ítems de análisis de contenido de guías docentes en materia de igualdad de género se muestran a continuación.

Tabla 1.

Instrumento para el análisis de las guías docentes

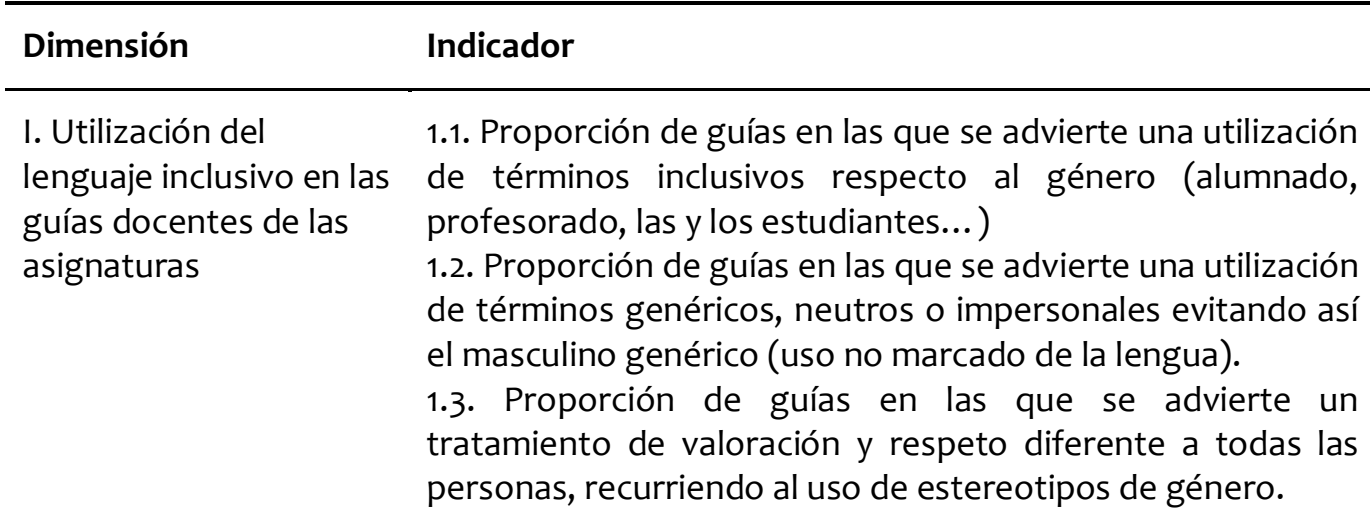

II. Inclusión de la perspectiva de género en las asignaturas y sus competencias, objetivos y contenidos.
2.1. Proporción de asignaturas obligatorias u optativas relacionadas con las mujeres y/o la igualdad de oportunidades entre mujeres y hombres.

2.2. Proporción de asignaturas obligatorias u optativas que cuentan con competencias transversales o específicas que aluden a la igualdad de género.

2.3. Proporción de asignaturas obligatorias u optativas que cuentan con competencias transversales o específicas que se relacionen con la igualdad de género de manera indirecta. 2.4. Proporción de asignaturas obligatorias u optativas que recogen temas de igualdad de género entre sus objetivos.

2.5. Proporción de asignaturas obligatorias u optativas que recogen temas de igualdad de género entre sus contenidos. 


\section{Dimensión Indicador}

III. Revisión de la bibliografía, recursos y materiales.
3.1. Tasas femenina y masculina de autoría entre las referencias bibliográficas. (¿Hay equilibrio o paridad entre el número de autoras $y$ autores en las referencias bibliográficas?)

3.2. Visibilización del género de las autoras o autores destacados en las asignaturas a través de la citación. (¿Se cita de tal manera que se permita visibilizar el género de las autoras y los autores destacados en las asignaturas?)

3.3. Presentación de materiales y recursos entre la bibliografía para el tratamiento de la igualdad de género en la práctica docente.

Por otro lado, para profundizar en el objeto de estudio y explorar las resistencias y realidades de las aulas respecto al tema objeto de estudio, se contactaron catorce profesores/as -cuatro hombres y diez mujeres- para, finalmente, realizar ocho entrevistas con académicas. Los profesores que respondieron, a pesar de reconocer la relevancia de este tipo de investigación, no accedieron a ser entrevistados argumentando motivos que van, fundamentalmente, desde la falta de tiempo a la falta de formación en materia de igualdad de género.

Las entrevistas semi-estructuradas en profundidad se realizaron a profesoras del grado de Educación Primaria, algunas de ellas miembros del equipo de gobierno de la facultad, del proyecto SUPERA que promueve la igualdad de género y del Seminario Interdisciplinar de Género y Educación (SIGE). Se ha recurrido a este tipo de entrevista ya que se entiende que las personas entrevistadas son sujetos activos y se les otorga la oportunidad de profundizar, aclarar, matizar, evaluar, alterar el orden, etc. A través de las entrevistas se trata de conocer cuestiones tales como la relevancia otorgada a la formación inicial en igualdad de género en la Facultad de Educación, el progreso que se ha dado en ella, las resistencias que existen y las posibilidades de mejora.

\section{Análisis y discusión}

\section{Análisis del contexto}

Para ofrecer un análisis contextualizado sobre la formación en igualdad de género se ofrecen datos segregados por sexo que aportan una visión global de la realidad estudiada. La Facultad de Educación cuenta con 192 mujeres pertenecientes al colectivo PDI (56,8\%) y 146 hombres (43,2\%) mientras que las personas catedráticas son 7 mujeres $(43,7 \%)$ y 9 hombres (56,3\%). En cuanto al alumnado de Grado, hay 3.062 mujeres (82,3\%) y 658 hombres $(17,7 \%)$ lo cual supone un total de 3.720 personas matriculadas. Los cargos de representación del profesorado se distribuyen, asimismo, de la siguiente manera: el decano es un hombre, la secretaria académica una mujer, las personas responsables de títulos son siete hombres y cinco mujeres y las vicedecanas son cinco frente a un vicedecano.

En el caso del Grado en Maestro en Educación Primaria la cifra es del 66,9\% de mujeres ( 827 frente a 409 hombres). Si se atiende a las áreas de especialidad que se ofrecen en este grado se puede apreciar como en la especialidad de Audición y Lenguaje las mujeres representan un $82,2 \%$ del alumnado matriculado, en la especialidad de Inglés un $81,3 \%$, en la 
especialidad de Música un 71,1\%, en la especialidad de Pedagogía Terapéutica un 91,3\% y en la especialidad de Educación Física un 42,9\%.

Tabla 2.

Tasas de mujeres y hombres según especialidad en el grado de Maestro en Educación Primaria (curso 2018-2019)

\begin{tabular}{lccc}
\hline Especialidad & Mujeres & Hombres & Porcentaje de mujeres \\
\hline Audición y lenguaje & 74 & 16 & $82,2 \%$ \\
\hline Inglés & 48 & 11 & $81,3 \%$ \\
\hline Música & 42 & 17 & $71,1 \%$ \\
\hline Pedagogía Terapéutica & 106 & 10 & $91,3 \%$ \\
\hline Educación Física & 98 & 130 & $42,9 \%$ \\
\hline
\end{tabular}

\section{Análisis de las guías docentes}

En el Plan de Igualdad aprobado por la Universidad Complutense de Madrid en el año 2015 se establecen como acciones en base a la legislación y el compromiso de la Universidad Complutense de Madrid promover, por un lado, un lenguaje inclusivo y no sexista en la comunicación y, por otro, prestar especial atención a la formación en igualdad en las titulaciones universitarias de Educación Infantil, Primaria y profesorado de ESO, Bachillerato y FP (Unidad de Igualdad, 2015).

Respecto al uso del lenguaje, el primer aspecto a señalar es el nombre oficial del grado denominado como "Grado en Maestro de Educación Primaria". Desde la universidad se hace uso de manera oficial de un lenguaje no inclusivo en un grado específico en el que las mujeres suponen un $66,9 \%$ del alumnado.

De igual forma, en tan solo 6 de las 72 de las asignaturas se observa un esfuerzo por utilizar lenguaje inclusivo (profesorado, alumnado, las y los estudiantes, etc.). En 36 guías se utiliza lenguaje no inclusivo, hablando en muy numerosas ocasiones de "el educador", "el profesor", "el niño", "los estudiantes" y "los maestros". Las 30 guías restantes no contienen un lenguaje que permita determinar si se trata de un lenguaje inclusivo en sí mismo, pero tampoco no inclusivo. En términos generales se identifica el uso del masculino para referirse tanto a profesorado como a alumnado de ambos sexos. A pesar del uso del lenguaje anteriormente mencionado, no se advierte el uso de estereotipos de género en las guías docentes o un tratamiento discriminatorio a raíz de ellos. Esto es, no se hace referencia a roles, actitudes o comportamientos entendidos como propios de uno u otro sexo que genere desigualdades y discriminaciones.

Las seis que utilizan lenguaje inclusivo son las siguientes: Didáctica de las Competencias Orales en Lengua Extranjera (Inglés), Fundamentos y Didáctica de Geografía, Matemáticas y su Didáctica I, Didáctica de la Lectura y de la Escritura en la Lengua Extranjera (Inglés), TICs Adaptadas a la Educación Primaria y Formación para el Bilingüismo (Inglés). Dos de ellas son obligatorias y tres pertenecen al departamento de Didáctica de la Lengua y la Literatura (Español, Francés e Inglés). 
En lo que a las asignaturas respecta, en el Grado de Maestro de Educación Primaria no existe ninguna cuyo foco sea la igualdad de género de manera específica. En lo relativo a las competencias, la alusión más común y directa a la igualdad de género se hace a través de la competencia transversal número 3 ( $\mathrm{CT}_{3}$ ). Esta competencia no garantiza que se atienda a la perspectiva de género puesto que abarca cuestiones muy diversas y se halla presente en 21 asignaturas (11 obligatorias y 10 optativas que suponen un $29,16 \%$ del plan total de estudios).

CT3. Analizar de forma reflexiva y crítica las cuestiones más relevantes de la sociedad actual que afectan el: impacto social y educativo de los lenguajes audiovisuales, cambios en las relaciones de género e intergeneracionales, multiculturalidad e interculturalidad, discriminación e inclusión social y desarrollo sostenible.

Existen otras competencias que pueden relacionarse con la igualdad de género de manera indirecta: CT16 -fomentar la práctica del pensamiento social crítico-, CM6.1.2 -conocer los diferentes tipos de familias, estilos de vida y educación en el contexto familiar- y $\mathrm{CG}_{5}$ analizar la importancia de los factores sociales y su incidencia en los procesos educativos-. Estas otras competencias que podrían relacionarse en un segundo nivel están presentes en 6 asignaturas (8,33\%).

Únicamente tres de 72 asignaturas (4,16\%) contienen el género entre sus objetivos o contenidos; solo una es obligatoria. La única asignatura obligatoria, Organización y Gestión de Instituciones y Programas Educativos, se cursa en primer curso y pertenece al Departamento de Didáctica y Organización Escolar. Recoge entre sus objetivos "enseñar y regular espacios de aprendizaje en contextos de diversidad que atiendan a las singulares necesidades educativas de los estudiantes, a la igualdad de género, a la equidad y al respeto a los derechos humanos" y "conocer y respetar las Leyes siguientes: Ley 3/2007, de 22 de Marzo, para la igualdad efectiva de mujeres y hombres; Ley 51/2003, de 2 de diciembre, de igualdad de oportunidades, no discriminación y accesibilidad universal de las personas con discapacidad y Ley $27 / 2005$, de 30 de Noviembre, de fomento de la educación y cultura de la paz". Además, entre sus contenidos, dentro del Bloque Temático II: La organización institucional explicita la "atención a la diversidad (de capacidades, de intereses, afectivosexual, de religión, etnia, clase, por incorporación tardía al sistema)".

La segunda asignatura, Aspectos Didácticos de la Educación Inclusiva, es optativa de segundo curso y también pertenece al Departamento de Didáctica y Organización Escolar. Esta asignatura incluye el objetivo de "diseñar y regular espacios de aprendizaje en contextos de diversidad que atiendan a las singulares necesidades educativas de los estudiantes, a la igualdad de género, a la equidad y al respeto a los derechos humanos". La tercera asignatura, Educación Física de Base es optativa de tercer curso y pertenece al Departamento de Expresión Musical y Corporal. Ésta tiene el objetivo de "ser capaces de identificar, clasificar y elaborar tareas adecuadas a cada uno de los contenidos estudiados, fomentando la igualdad de género".

Al igual que sucede con las competencias, entre los objetivos y contenidos también es común que se realicen referencias a las desigualdades sociales, pero no a las de género concretamente. No aparecen contenidos relativos al papel de las mujeres ni se hace mención a las mujeres en la Historia o a sus limitaciones de acceso a ciertos ámbitos y a los roles sociales relevantes. Asimismo, la guía del Trabajo de Fin de Grado no incluye entre sus posibles temas uno específico de género. Tampoco en la guía de las prácticas curriculares se 
especifica alguna competencia a desarrollar durante las mismas relativa al género. De las 72 asignaturas que componen el total del plan de estudios de Educación Primaria, 51 (70, 83\%) no hacen referencia a la igualdad de género en ninguno de los apartados de sus guías. Se comprueba así que no existe una aplicación real de la legislación vigente y la existencia de una clara brecha entre la teoría y la práctica docente en la formación inicial del profesorado de Educación Primaria.

Por último, al revisar la bibliografía, recursos y materiales, Barbieri (2013) citado en Ros et al. (2016) plantea la necesidad de "recuperar las aportaciones de las mujeres en la sociedad y la cultura para hacerlas visibles en la creación (científica en nuestro caso), la vida cotidiana y la historia". Por ello, se presenta como objeto de análisis el apartado "referencias bibliográficas" que permite conocer, entre otras cosas, las contribuciones que se recogen de las mujeres para la formación inicial del profesorado.

Tabla 3.

Número de mujeres y hombres autoras de la bibliografía de las guías docentes

\begin{tabular}{lccc}
\hline Curso & Mujeres & Hombres & Porcentaje de mujeres \\
\hline Primero & 28 & 114 & $19,7 \%$ \\
\hline Segundo & 138 & 217 & $38 \%$ \\
\hline Tercero & 91 & 186 & $32 \%$ \\
\hline Cuarto & 61 & 91 & $40 \%$ \\
\hline
\end{tabular}

En el primer curso hay un total de 28 mujeres entre las 142 personas autoras $(19,7 \%$ de mujeres). En el segundo curso aparecen un total de 138 mujeres entre 355 personas autoras (38\% por ciento de mujeres). En el tercer curso existen un total de 91 mujeres entre 277 personas autoras (32\% de mujeres) y en el cuarto curso hay un total de 61 mujeres entre las 152 personas autoras (40\% de mujeres). Resultan especialmente llamativas dos asignaturas: por una parte, Sociología de la Educación, que no recoge a ninguna mujer entre 16 referencias y 22 autores y, por otra parte, Historia de España y su Didáctica, que contiene en su bibliografía un libro relativo a la historia de las mujeres en España y América Latina.

En los apartados de referencias se observa una tendencia a utilizar la inicial de los nombres de las autoras y autores, invisibilizando así el género de la persona. Finalmente, no se aportan recursos electrónicos tales como webs o blogs con contenidos sobre igualdad de género que puedan facilitar la práctica docente entendida desde la perspectiva de género.

\section{Análisis de las entrevistas}

En este tercer apartado se expone el análisis de las entrevistas poniendo énfasis en los aspectos que dificultan o impiden la formación inicial del profesorado en igualdad de género. Las informantes clave consideran que la existencia de menciones de especialización, una formación práctica que se amplía con el paso de los cursos y un grupo bilingüe (inglés-castellano) han supuesto una mejora en el Grado de Maestro en Educación Primaria. Aun así, mantienen una visión crítica de la educación y entienden que la formación del profesorado es insuficiente.

"Mi visión es que, desde lo que he recibido de los estudiantes y lo que compartimos los compañeros y compañeras, las asignaturas disciplinares no enseñan cómo enseñar, sino que enseñan los contenidos en sí mismos [...] Creo que está muy bien 
la formación del prácticum, pero la idea que me transmite el alumnado es que aún no saben muy bien cómo dar algunos contenidos. (Profesora PSHI4)

Argumentan que la formación no responde a las necesidades actuales de las aulas. Esto surge de la inmovilidad de los planes de estudios desde su creación y acreditación por parte de la Agencia Nacional de Evaluación de la Calidad y Acreditación (ANECA) hace más de una década. Las asignaturas apenas han variado desde que se creó el grado en el año 2007 y la introducción de las menciones, el único gran cambio de las titulaciones, se realizó a través del El Real DECRETO 1594/2011, de 4 de noviembre, que retoma las especialidades docentes. Por ello, identifican como debilidades en el grado no solo una formación basada en contenidos que el alumnado ha de aprender, sino también otras cuestiones tales como la falta de formación en igualdad de género o el desarrollo del pensamiento crítico: "La formación de los maestros no responde a lo que debería ser la formación del magisterio porque no aporta las nuevas tendencias, la innovación... No hay una renovación educativa o planteamientos novedosos". (Profesora PSSU6)

Insisten en la carencia de asignaturas, competencias e incluso objetivos relativos a la igualdad de género como una de las cuestiones deficientes en la formación del grado. A pesar de que exista una competencia transversal $\left(\mathrm{CT}_{3}^{3}\right)$ que haga referencia al análisis reflexivo y crítico de los cambios en las relaciones de género, entienden que la introducción de este aspecto únicamente de manera transversal a día de hoy no garantiza que se forme adecuadamente a las futuras maestras y maestros puesto que no todo el profesorado comprende la importancia de la perspectiva de género o cuenta con la formación suficiente para introducirla de manera transversal en su docencia. Además, es una elección personal formarse ya que las opciones de formación permanente son voluntarias.

"La formación transversal ahora mismo está en terreno de nadie. Si yo ya tengo en mi programa que dar ciertas cosas, ¿también voy a dar igualdad de género? Además, ¿si no estoy formado? Lo transversal con currículums cargados de contenidos conceptuales se queda en nada. Tiene que ser obligatorio y evaluable". (Profesora PDIV1)

"Hay cursos que se hacen si quieres y que, al final, la realidad de esos cursos es que va quien menos lo necesita. Los cursos de identidades, de diversidad sexual... salen anualmente; o sea, que hay formación, lo que pasa es que es formación voluntaria". (Profesora PDIV1)

Todas consideran que la formación permanente debería ofrecerse en la universidad de manera gratuita, que se le habría de dar visibilidad y que podría incentivarse. Sin embargo, difieren en si debería ser obligatoria o voluntaria. Mientras que algunas opinan que no puede obligarse a las personas porque sería contraproducente, otras consideran que debería ser obligatorio porque ha de ser algo transversal al tratarse de una cuestión fundamental en la enseñanza.

\footnotetext{
${ }^{3}$ CT3. Analizar de forma reflexiva y crítica las cuestiones más relevantes de la sociedad actual que afectan al impacto social y educativo de los lenguajes audiovisuales, cambios en las relaciones de género e intergeneracionales, multiculturalidad e interculturalidad, discriminación e inclusión social y desarrollo sostenible.
} 
"Si se pudiera hacer una política de formación permanente del profesorado sería muy positivo [...] el poner obligatorio es algo muy complicado, pero en esta facultad debería serlo... igual que hacemos cursos de campus virtual para manejar una herramienta hoy día deberíamos tener la obligatoriedad de haber recibido una formación básica sobre género. Estamos formando a maestros y maestras y hay personas que tenemos formación, pero aquellas que no la tienen deberían hacerla". (Profesora PSSU6)

En cuanto a por qué no se introduce formación específica en igualdad de género, las informantes no hablan de resistencias institucionales. Al contrario, consideran que se está dando un especial apoyo a las propuestas y se están promoviendo iniciativas como la Unidad de Igualdad de la UCM o el uso inclusivo del lenguaje en la Facultad de Educación y la universidad al completo. Además, no piensan que pueda retrocederse con los avances conseguidos en la facultad puesto que se está procurando que los cambios que se introduzcan no sean únicamente superficiales.

"Para conseguir que una persona hable de cuestiones de género en su aula lo primero tienes que hacer ver la necesidad. A nivel institucional estamos tratando de visibilizarlo a través del lenguaje en mensajes, cartas, carteles... Porque eso se va incluyendo en la cultura de la organización". (Profesora PDES7)

Tampoco entienden que existan resistencias entre el profesorado universitario que afecten de manera significativa a la introducción de la perspectiva de género en la formación inicial del profesorado. Se ha dado un progreso con el paso de los años que ha permitido que las resistencias iniciales hayan mermado y a día de hoy no supongan un obstáculo frontal y directo. Además, cada vez con más frecuencia el profesorado se implica en propuestas relacionadas con la igualdad de género. Reconocen que todavía pueden existir resistencias individuales, sobre todo por desconocimiento de la igualdad de género, pero que no suponen un impedimento para llevar a cabo actividades o proyectos que se presentan.

"Al comenzar el Seminario Interdisciplinar Género y Educación hace doce años algunas personas nos dijeron que éramos el 'lobby feminista' e incluso cuando hemos solicitado alguna ayuda nos la han negado por decir que eso no era pertinente pero poco a poco, además de que hay más conciencia social, la gente se ha ido sumando. La sensación que tengo es de que cada vez hay más conciencia, pero sigue sin ser una línea prioritaria”. (Profesora PSSI5)

Destacan que, aunque no consideren que existan resistencias determinantes entre el profesorado de manera general, éste continúa sin incluir la perspectiva de género en sus clases. El nivel de concienciación y de implicación no es el deseable entre el profesorado de una facultad tan relevante como es la de Educación y Formación del Profesorado. Sienten que existen defectos en la comunicación interna a nivel de facultad y que el profesorado universitario no cuenta con recursos y tiempo para dedicar a este tipo de cuestiones de manera extraoficial. Esto fomenta que no se lleven a cabo iniciativas amparadas por el claustro de manera general, algo indispensable para introducir eficazmente la perspectiva de género en la universidad. 
"No se trata únicamente de reivindicar lo mal que estamos. Muchas veces venimos a la facultad y nos dejamos llevar, y ese es uno de los problemas que pueden existir. Hay cuatro personas que están a tope y el noventa y nueve restantes se dejan llevar". (Profesora PDES7)

Las entrevistadas perciben una situación similar por parte del alumnado en cuanto a resistencias. No hacen alusión a resistencias que supongan un impedimento para el desarrollo normal de las asignaturas cuando se tratan cuestiones de igualdad de género, pero sí han detectado en ocasiones resistencias individuales. Identifican más resistencias en grupos formados mayoritariamente por hombres, fundamentalmente en la especialización de Educación Física en la que detectan un mayor desconocimiento y desinterés inicial hacia las cuestiones relativas a la igualdad de género. Aun así, su experiencia refuerza el argumento sobre la necesidad de la formación del alumnado puesto que las resistencias disminuyen con el desarrollo de las asignaturas.

"En esta facultad, por suerte, he sentido rechazo cuando yo planteo temas de género o cuando hablo de feminismo en casos muy puntuales. Más dudas que rechazo. Normalmente, en los grupos de Educación Física, supongo que por temas de estereotipos y de socialización es más difícil trabajar estos temas, pero cuando lo planteas y lo trabajas se avanza". (PSIN3)

Para finalizar, defienden que lo óptimo sería introducir una asignatura concreta de carácter obligatorio en igualdad de género en todos los grados y, de manera específica, en aquellos relacionados con la educación. Piensan que el único modo de asegurar una formación real es incluir en el currículum una materia de carácter obligatorio que sea evaluable y se componga de créditos ECTS al igual que el resto de las asignaturas. A pesar de ello, reconocen la dificultad de introducir materias nuevas en el plan de estudios existente ya que ha de ser la ANECA quien apruebe las modificaciones que se realicen sobre el plan de estudios, y eso solo sería posible si se contara con un consenso y empeño colectivo ya que una transformación profunda supondría una gran carga de trabajo. Por ello, consideran una opción más viable incluir una asignatura optativa de manera previa a la modificación de la estructura del grado al completo: "Es tan complejo que... lo tendremos que hacer en algún momento, pero el día que se haga va a requerir muchísimo trabajo y muchísimo esfuerzo que ahora mismo no sé si somos capaces de afrontar". (Profesora PSPS2)

\section{Conclusiones}

Incorporar la perspectiva de género en la formación inicial del profesorado de Educación Primaria resulta una necesidad actualmente no solo para construir una escuela democrática e igualitaria, sino también para cumplir con la legalidad establecida en el sistema educativo español. El profesorado de Educación Primaria tendrá en sus manos la educación de muchas niñas y niños que formarán la futura ciudadanía, lo cual les confiere un poder de cambiar la sociedad o mantener los patrones y estereotipos actuales que reproducen desigualdades entre hombres y mujeres.

A pesar de la evidente feminización de la Facultad de Educación, en todos sus niveles y agentes, la denominación del grado "Maestro en Educación Primaria" afianza la idea de que el lenguaje continúa siendo androcéntrico. De igual forma, el análisis de las guías docentes pone de manifiesto un claro déficit en el uso del lenguaje inclusivo, la inclusión de la 
igualdad de género entre los contenidos, objetivos y competencias, así como una invisibilización de las aportaciones de las mujeres a la historia y el conocimiento científico.

A través de las entrevistas se observa la necesidad de mejorar a la formación inicial del profesorado, lo cual pasa por modificar los planes de estudios. El profesorado universitario no muestra interés a este respecto a pesar de que la formación permanezca desconectada de la realidad actual. Una de las mayores resistencias identificadas es la falta de recursos para dedicar una parte del tiempo a cuestiones ajenas a la investigación y la docencia. Además, la falta de formación en igualdad de género supone que una gran parte del profesorado no identifique esta cuestión como relevante y no dedique tiempo y esfuerzo a un proyecto de gran envergadura como es la modificación de los planes de estudios.

Finalmente, se puede intuir cómo sí supone una dificultad o resistencia el hecho de que la ANECA no cumpla con su labor de revisar y asegurar la inclusión de asignaturas en igualdad de género y no ponga los medios necesarios para que se cambien los planes de estudios. Esto hace que el profesorado se ampare en esta deficiencia para no implicarse en ello. A pesar de que la institución universitaria avanza hacia la igualdad de género, no existe una transformación realmente significativa que únicamente se conseguirá cuando se modifiquen los planes de estudios y se introduzca, de manera oficial y real, la perspectiva de género en la formación inicial y permanente del profesorado.

\section{Referencias}

Asián, R., Cabeza, F. y Rodríguez, V. (2015). Formación en género en la universidad: ¿materia de asignaturas específicas o de educación transversal? Revista historia de la educación latinoamericana, 17 (24), 177-183

Azorín, C. (2015). Análisis de la política de género en España, hacia una educación inclusiva y coeducadora. III Congreso Internacional de Investigación e Innovación en Educación

Infantil y Educación Primaria. Murcia. Lugar de publicación: http://congresos.um.es/coni3p/coni3p2015/paper/view/36311

Ballarín, P. (2001). La educación de las mujeres en la España contemporánea (siglos XIX-XX). Madrid: Síntesis

Ballarín, P. (2008). Guía de buenas prácticas para favorecer la igualdad entre hombres y mujeres. Consejería de Educación: Junta de Andalucía.

Bourdieu, Pierre. y Passeron, Jean Claude. (1996): La reproducción: elementos para una teoría del sistema de enseñanza. México: Fontamara.

Colectivo Feminario de Alicante. (1987). Elementos para la educación no sexista. Víctor Orenga, Editores: Valencia.

Díaz de Greñu, S., y Anguita, R. (2017). Estereotipos del profesorado en torno al género y a la orientación sexual. Revista electrónica interuniversitaria de formación del profesorado, 20 (1), 219-232.

Gómez, I. (2015). Formación del profesorado para el tratamiento educativo de los conflictos sobre diversidad cultural y de género. (Tesis doctoral). Universidad Complutense de Madrid, Madrid.

Gómez, I. y Sánchez, P. (2017). Formación del profesorado en cuestiones de género. Revista de Comunicación de la SEECl, 43, 53-68. 
González, T. (2017). Políticas educativas igualitarias en España. La igualdad de género en los estudios de magisterio. Archivos Analíticos de Políticas Educativas, 26 (2). http://dx.doi.org/10.14507/epaa.26.2764

Ortega, D. y Pagés, J. (2018). Género y formación del profesorado análisis de las guías docentes del área de didáctica en las ciencias sociales. Contextos educativos: Revista de educación, 21, 53-66.

Rebollo, M. J. (2006). El currículum en la escuela coeducativa. La educación de las mujeres y niñas en Iberoamérica, 151-174.

Ros, R. D., Hernández, B. M. A., Trigueros, I. M., Cabezas, I. L., Martínez, M. I., Castelló, T. D. P., y Vera-Muñoz, M. I. (2016). Inclusión de la perspectiva de género en las guías docentes de asignaturas de Didáctica. En XIV Jornadas de Redes de Investigación en Docencia Universitaria: Investigación, innovación y enseñanza universitaria: enfoques pluridisciplinares (pp. 2665-2679). Instituto de Ciencias de la Educación.

Subirats, M. (1991). La educación como perpetuadora de un sistema de desigualdad: la transmisión de estereotipos en el sistema escolar. Infancia y Sociedad: revista de estudios, 10, 43-52.

Unidad de Igualdad. (2015). Plan de Igualdad. Universidad Complutense de Madrid. Recuperado de https://www.ucm.es/data/cont/media/www/pag3331/PLAN\%20DE\%20IGUALDAD.pdf

Universidad Complutense de Madrid. Facultad de Educación - Centro de Formación del Profesorado. Guías docentes de las asignaturas. Recuperado de https://educacion.ucm.es/estudios/grado-educacionprimaria-plan

Varela, N. (2014). Feminismo para principiantes. B DE BOOKS: Madrid

\section{Legislación}

Constitución Española. Boletín Oficial del Estado, 29 de diciembre de 1978, núm. 311, pp. 29313 a 29424

DECRETO 32/2017, de 21 de marzo, del Consejo de Gobierno, por el que se aprueban los Estatutos de la Universidad Complutense de Madrid.

DECRETO 89/2014, de 24 de julio, del Consejo de Gobierno, por el que se establece para la Comunidad de Madrid el Currículo de la Educación Primaria. Vicepresidencia, Consejería de Presidencia y Portavocía del Gobierno. Comunidad de Madrid.

Ley $2 / 2016$, de 29 de marzo, de Identidad y Expresión de Género e Igualdad Social y no Discriminación de la Comunidad de Madrid. Boletín Oficial del Estado, nº 98, 2016, 26 abril.

Ley General de Educación y Financiamiento de la Reforma Educativa (LGE). (Ley 14/1970, de 4 de agosto). Boletín General del Estado, nº 186, 1970, 6 agosto.

Ley Orgánica de Calidad de la Educación (LOCE). (Ley Orgánica 10/2002, de 23 de diciembre). Boletín Oficial del Estado, $\mathrm{n}^{\circ}$ 307, 2002, 24 diciembre.

Ley Orgánica de Educación (LOE). (Ley Orgánica 2/2006, de 3 de mayo). Boletín Oficial del Estado, $n^{\circ}$ 106, 2006, 4 mayo. 
Ley Orgánica de Medidas de Protección Integral contra la Violencia de Género. (Ley Orgánica 1/2004, de 28 de diciembre). Boletín Oficial del Estado, n³13, 2004, 29 diciembre.

Ley Orgánica de Ordenación General del Sistema Educativo (LOGSE). (Ley Orgánica 1/1990, de 3 de octubre). Boletín Oficial del Estado, n² 295, 1990, 21 noviembre.

Ley Orgánica para la igualdad efectiva de mujeres y hombres (Ley Orgánica 3/2007, de 22 de marzo). Boletín Oficial del Estado, $n^{\circ}$ 71, 2013, 27 julio.

Ley Orgánica para la Mejora de la Calidad Educativa (LOMCE). (Ley Orgánica 8/2013, de 9 de diciembre). Boletín Oficial del Estado, nº 295, 2013, 10 diciembre. 